\section{Hemispheric mediation of same-different judgments: A reply}

DAVID B. BOLES

Rensselaer Polytechnic Institute, Troy, New York

\author{
SEBASTIANO BAGNARA \\ Institute of Psychology, CNR, Rome, Italy \\ FRANCESCA SIMION \\ University of Padua, Padua, Italy \\ and

\section{CARLO UMILTÀ University of Parma, Parma, Italy}

Bagnara, Boles, Simion, and Umiltà (1983) reported that, in matching letters for physical identity, "same" responses are made more quickly than "different" responses, regardless of the delay between the letters in a pair. This was eliminated, however, when asymmetric letters were considered and when "different" pairs were made sufficiently dissimilar in visual terms. At the same time, the type of response interacted significantly with visual field, such that there existed tendencies for "same" responses to be made more quickly and with fewer errors in the right visual field, while "different" responses were faster and more accurate in the left visual field.

Bagnara et al. argued that these results were incompatible with several current models of letter matching. The observed effects of similarity and symmetry were seen as difficult to reconcile with the idea that matching same-case pairs involved a "name" code (Proctor, 1981), and the observation that samedifferent disparity existed when there was no delay between letters argued against the priming model of letter matching (Proctor, 1981). Similarly, the effects of symmetry were viewed as inconsistent with a single process model employing a "difference counter" (Krueger, 1978). The visual field effects, on the other hand, were taken as arguing against a hemispheric model stating that "same" and "different" responses rested on right-hemisphere holistic and left-hemisphere analytic processes, respectively (a view that emerges in combining the letter-matching work of Taylor, 1976, and others, with the hemispheric work of Patterson \& Bradshaw, 1975, and others). Two other hemispheric models (Hellige \& Webster, 1979; Patterson \& Bradshaw, 1975) predicted visual field $x$ similarity interactions, which were not obtained

Reprint requests should be addressed to David B. Boles, Department of Psychology, Rensselaer Polytechnic Institute, Troy, NY 12181. by Bagnara et al., so these were also seen as difficult to reconcile with the results.

Since the empirical results did not accord well with extant models, we attempted to outline a model that could at least account for the results we obtained. This incorporated certain desirable features of the models we had rejected, including the notion of dual processing (Bamber, 1969; Hock, 1973; Taylor, 1976), since it was necessary to account for effects of both display symmetry (which can affect only "same" responses) and different pair similarity (which affects largely, though not exclusively, "different" responses). A distinction was drawn, however, between dual processing as traditionally proposed (holistic vs. analytic) and the notion of dual processing that our results supported (superfeatural vs. featural). We explicitly rejected the former and accepted the latter, defining a superfeatural process as one that extracts symmetry information both within and between letters. A featural process was taken as implying an analytic process operating at the feature level. Another aspect of the proposed model was that it incorporated a criterial assumption (Nickerson, 1981) and a "difference counter" operating to produce "different" responses (Krueger, 1978). With these assumptions, the model could account for all of our major results, with the exception of the visual field effects, which were viewed as somewhat inexplicable though not without precedent (Bagnara, Boles, Simion, \& Umiltà, 1982; Cohen, 1973; Egeth \& Epstein, 1972).

In reply to our paper, Sergent (1984) advances a number of criticisms she believes reduce the validity of our arguments. She takes to task our failure to control viewing distance, on the grounds that this introduces unnecessary error into the experiments. She also notes that response hand was not analyzed, with the consequence that hand $\times$ visual field $\times$ match interactions cannot be assessed. Third, she views the dual-process view of letter matching as inherently problematic, reducing its value as a model. Fourth, she sees symmetry as irrelevant to the dual-process model, in part because symmetry may also affect "different" responses. Fifth, Sergent notes that there may be isomorphism in the predictions made by holistic and analytic processes with respect to the effects of similarity. And, finally, she claims that the visual field $\times$ match interaction, on which our rejection of the holistic-analytic hemispheric model is based, is not consisent in our experiments or in the literature. We will now address these criticisms in turn.

\section{Viewing Distance}

The gist of the viewing distance criticism is that allowing subjects to view the display at self-selected distances may preclude finding visual field effects. 
While literature can be cited for both sides of the argument relating to variation in eccentricity (see Bagnara et al., 1983, and Sergent, 1984, for references) and stimulus size (see Sergent, 1984, and Boles, in press, for references and experimental results), the issue seems to come down to whether the lack of control over distance introduced sufficient error variance to rule out significant field differences. This is an empirical question answerable with the obtained data.

Perhaps the strongest argument against the criticism is that visual field effects were obtained. In Experiment 1, field interacted significantly with the type of match in both RT $(p<.05)$ and errors $(p<$ .05 ). The same was true in Experiment 2 for $R T$ $(p<.025)$. Since this was one of two critical interactions bearing on hemispheric models of letter matching, and it involved the visual field variable, it is clear that the method used had sufficient precision to assess the field effects under consideration. The fact that the interaction was opposite in direction to the one predicted is, of course, irrelevant to the precision question.

The other critical interaction was that of visual field $x$ similarity, which was not significant in any analysis. That the method used had sufficient precision to detect this interaction is implied indirectly by the field $\times$ match outcomes, but we can also report power analyses (Keppel, 1973) that bear directly on the question. Thus, in Experiment $1 \mathrm{RT}$, there was .80 power to find a field $\times$ similarity interaction of $26 \mathrm{msec}$, with two-tailed alpha $=.05$. The corresponding precision in Experiment 2 was for an interaction of $12 \mathrm{msec}$. We believe that these figures represent a substantial degree of precision. This is particularly true for Experiment 2, but in both cases the outcomes are within the sizes of the significant field $X$ match interactions (32 and $19 \mathrm{msec}$, respectively). While we would not advise a repetition of our procedure in letting subjects self-select viewing distances, and in retrospect agree with Sergent that it is desirable to be able to specify a methodological parameter, we conclude that variation in viewing distance has little implication for our results.

\section{Response Hand}

Sergent argues that, to provide information on possible interaction with other factors, response hand should have been included in our analyses. In our methodology, subjects in each experiment were assigned to one of two groups, either with the left hand responding "same" and the right hand responding "different," or vice versa. Since we recorded these assignments, we can now report the effect of response hand. Figures 1 and 2 show the visual field $\times$ match $\times$ response hand interactions for Experiments 1 and 2, respectively, these being the

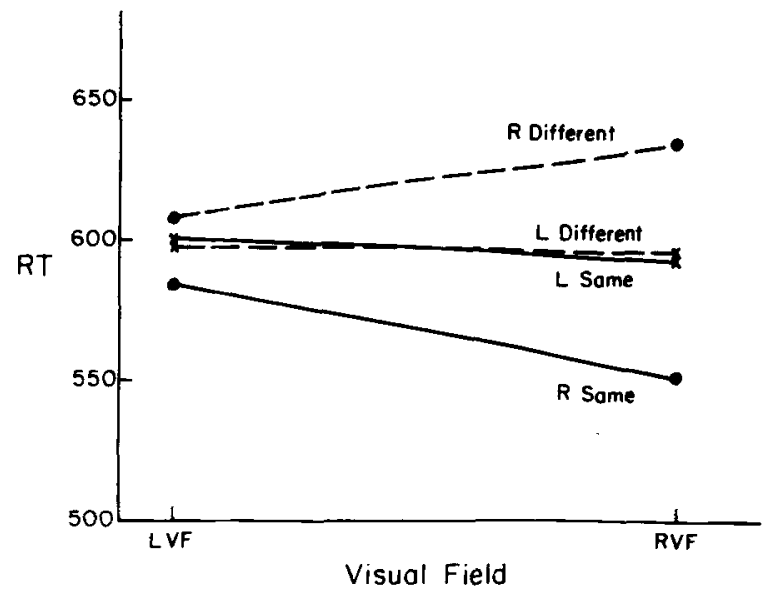

Figure 1. Visual field $\times$ match $\times$ response hand interaction in Experiment 1 of Bagnara et al. (1983).

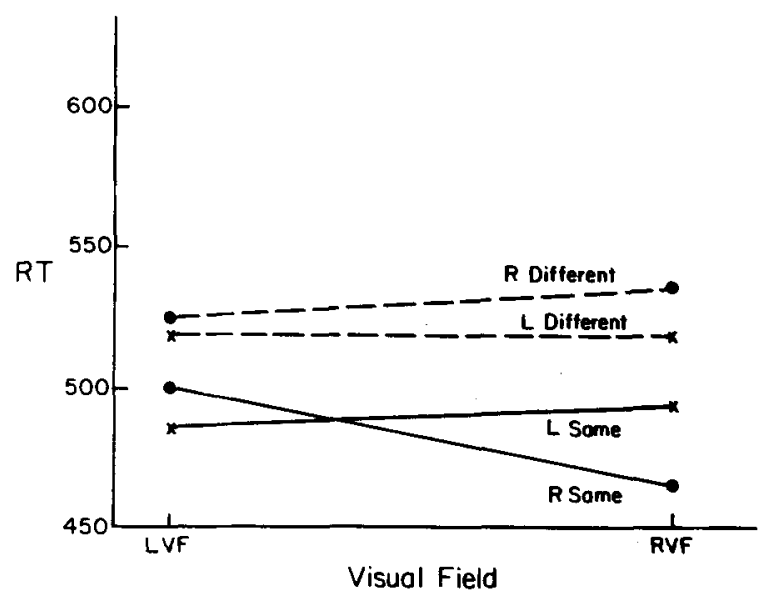

Figure 2. Visual field $x$ match $\times$ response hand interaction in Experiment 2 of Bagnara et al. (1983).

interactions mentioned by Sergent as being of greatest interest.

We should first point out that, because of structural problems in the data, we have not attempted to assess significance levels for these interactions: within each subject, the type of response is confounded with response hand, so, although all three variables are within-subject, the data cannot be structured into a within-subject analysis of variance. Nevertheless, it must be conceded that the type of interaction proposed by Sergent is present in both experiments. That is, the field $\times$ match interaction exists only for the right hand and not for the left hand, as is evident on inspection of Figures 1 and 2.

Although impressed by the prescience of the prediction of the interaction, we are skeptical about the 
implications for our arguments. It simply is not clear why such an interaction should be obtained (Jonides, 1979), prompting us to repeat our statement that more research is needed to discover the exact reasons for obtaining such results. One thing that is quite clear, however, is that there is little comfort in these outcomes for those who would endorse the view that "same" responses involve a right-hemisphere holistic process and "different" responses, a left-hemisphere analytic process. Our visual field $\times$ match interactions were originally presented as inconsistent with this view, and the three-way interaction is no more consistent. Furthermore, the three-way interaction shows that somewhat different processes must underlie "same" and "different" matches, or there would be no interaction with hand and visual field. Thus, although the picture has been complicated a little, our original conclusions remain intact.

\section{The Dual Process View}

Sergent argues that there is a problem inherent in the view that dual processes underlie letter matching, that is, the dual processing involves strategies that must be implemented at the outset, before letter comparison takes place. This is presented as one reason why the acceptance of a dual process view by Bagnara et al. is uncompelling.

In our opinion, this argument is naive and ignores the intent of dual process models of letter matching. The existence of dual processes was proposed historically not to account for strategic effects, but to account for same-different effects found in mixed blocks of trials when the subject in fact never knows what type of pair will be presented next (Hamber, 1969; Taylor, 1976). Both processes were assumed to proceed in parallel, with the holistic one being the faster of the two. In this way, according to the model, "same" responses are typically faster than "different" responses. Thus, dual-process models emerged from an experimental milieu in which strategy was irrelevant due to mixed block design, and in which the orientation was toward parallel processes emerging at different times.

Nevertheless, although it is important to understand exactly what the dual process model says, Sergent erroneously identifies our theoretical position with the model. It should be clear to most readers that we, in fact, rejected it in favor of a superfeaturalfeatural model, stating explicitly toward the end of our paper that "a holistic-analytic model no longer appears tenable."

\section{Symmetry Effects}

Sergent argues that, since we did not examine symmetry effects for "different" pairs, but only for "same" pairs, we cannot validly draw conclusions with respect to a dual-process model. This criticism, we concur, is well taken for the effects of letter sym- metry, referring to the existence of visual symmetry within a letter (e.g., A or C). However, the argument has no validity with respect to display symmetry, referring to symmetry across letters (e.g., AA but not CC). Display symmetry can exist only for "same" pairs, so, to the extent that responses are affected by it, there exists de facto evidence for a process underlying "same" matches which does not underlie "different" matches. We reported a significant effect of display symmetry, teased out from letter symmetry by using both horizontal and vertical displays. Consequently, we believe that the display symmetry effect is valid evidence for a process affecting only "same" pairs.

\section{Isomorphism}

Sergent argues that holistic and analytic processes are isomorphic with respect to predicting the effect of similarity on different pairs. The analytic model predicts that increased similarity will lengthen RTs due to the difficulty the analytic process has in finding a feature that differentiates the two letters. The holistic model, according to this line of criticism, also predicts lengthened RTs, but this is due to overall similarity between the letters, which makes their global forms more similar. By this argument, the holistic and analytic models are considered inseparable.

When taken in isolation, this argument is quite plausible; what should not be forgotten is that the effect of similarity is only one of the predictions made by these processes. There is at least one other. This is that "same" responses should be faster than "different". responses, assuming that a holistic process is faster than an analytic process (Taylor, 1976). To the extent that this is shown to be incorrect, the dualprocess model is in question.

What we, in fact, achieved was to show that when "different" pairs are sufficiently discriminable, and asymmetric letters are considered, the same-different disparity essentially disappears. This would seem to throw the dual-process model into question. As a consequence, we rejected the model.

Although this outcome suggests that the two processes are not completely inseparable, the thrust of Sergent's criticism is that, in her view, we have no reason to propose in our discussion section that "different" matches are made analytically. Presumably, both "same" and "different" matches could be made holistically. Again, this point is well taken when the similarity effect is considered in isolation, but there are two other reasons for proposing that an analytic process is involved. One is provided by the work by Krueger and his colleagues on the "difference counter" (Krueger, 1978; Shapiro \& Krueger, 1983). Its thrust is that the matching of letters involves tallying a count of their differences. If the system is noisy, as Krueger proposes, then positive counts can 
occur even when letters are identical, so that all nonzero counts must be rechecked for accuracy. This produces faster RTs for "same" matches than for "different" matches, since some "same" matches can be responded to quickly because of zero counts, while virtually all "different" matches involve rechecking nonzero counts.

In this context, it should be easier to understand why we have supported an analytic process as underlying "different" matches. A "different counter" is preeminently analytic, if by analytic we mean the comparison of features (whether serially or in parallel) rather than whole patterns. To the extent that it is supported by research, there is no alternative to positing an analytic process. In this particular instance, adoption of the model also makes sense of our similarity and symmetry effects. When pairs are made sufficiently dissimilar, rechecking is no longer needed, since, even if nonzero, the counts for "same" pairs would be much lower than for "different" pairs. When symmetry is not present, so that only asymmetric letters are considered, the proposed superfeatural process cannot operate, so the combined effects of nullifying this and the rechecking processes result in "different" responses being as fast as "same" responses. Thus, the combined pattern of results and its potential consistency with the "difference counter" is an important reason why we proposed that an analytic process underlies "different" matches.

Another result also pointed us in this direction, however. Bagnara et al. (1982) found that RT in a letter-matching task depended on a quantified index of the number of features within letters. Thus, block letters with few line segments (e.g., F and $\mathrm{H}$ ) produced faster RTs than block letters with relatively more line segments (e.g., E and A). Although this effect disappeared with practice, the fact that it existed at all seems more straightforwardly consistent with an analytic process than with a holistic process. In the case of an analytic process, if there are more features to compare between letters, then one might expect longer RTs. In the case of a holistic process, however, and in the absence of additional assumptions, the number of features should be irrelevant to RTs, since only global patterns, and not features, are compared.

In summary, a portion of our results contradicted the assumption of fast holistic and slow analytic processes underlying "same" and "different" matches, respectively, resulting in a rejection of the dualprocess model. We adopted the analytic process for "different" matches, however, because it was largely consistent with Krueger's work and our prior work, and because the model could explain certain obtained effects of similarity and symmetry.

\section{Visual Field by Match}

The final criticism concerns the robustness of the visual field $\times$ match interaction. Sergent argues that it is not a constant feature of our experiments or of the literature, and so therefore does not support the view of a general dissociation of match type $\times$ hemisphere.

We are actually a bit surprised by this criticism, because we thought we had outlined a similar position. We pointed out that, in these particular experiments, such interactions were obtained and that they seemed to support a process dissociation of the two types of matches (a view, we have argued above, that is complicated but not contravened by interaction with response hand). However, we were also careful to close with the observation that, although such interactions are not without precedent, the samedifferent factor also frequently fails to interact with visual field (Atkinson \& Egeth, 1973; Egeth, 1971; Moscovitch, Scullion, \& Christie, 1976). In our opinion, the proportion of times such interactions occur is not at issue. That they occur at all, however, is important, and in this regard we think it was legitimate to cite the sources we cited.

In any case, criticism about the modal result misses a crucial point. A hemispheric model stating that "same" matches are right-hemisphere holistic in nature, and that "different" matches are left-hemisphere analytic in nature, would predict a significant visual field $\times$ match interraction opposite the type we obtained. Even if the modal result is a null effect, as Sergent argues (and we concur), this hardly supports the model.

\section{Conclusions}

Of the six major criticisms raised by Sergent (1984) against the paper by Bagnara et al. (1983), we view none as having a great practical import. To recap, uncontrolled viewing distance, while perhaps an undesirable feature of the methodology, neither precluded finding visual field effects nor introduced insurmountable error variance into the results. Inclusion of response-hand effects showed that, although this variable modifies the obtained visual field $x$ hand interactions, it leaves intact the conclusions regarding dissociation of processes and inconsistency with one of the hemispheric models of letter matching. Sergent's a priori criticism of the dual-process model of letter matching is founded in two misunderstandings: first, the nature of the processes themselves, which have been proposed to emerge in parallel and not strategically, and second, a confusion of the holistic-analytic dual-process model with our proposed superfeatural-featural distinction. Symmetry effects are found not only within but between letters (within displays), constituting de facto evi- 
dence for some scheme of dual processing (such as superfeatural-featural). Holistic and analytic processes are not completely isomorphic in their predictions, but can be distinguished on the basis of same-different disparity, by work on the "difference counter," and by the effects on RT of a quantified index of the number of letter features. Finally, inexplicable visual field $x$ match interactions are occasionally obtained, frequent failures to find such interactions notwithstanding.

While we are therefore not impressed by Sergent's arguments, we welcome the opportunity afforded to debate several important issues in detail. This can only have a beneficial effect in the endeavor to arrive at a necessary and sufficient model of physical letter matching.

\section{REFERENCES}

Atkinson, J., \& Egeth, H. (1973). Right hemisphere superiority in visual orientation matching. Canadian Journal of Psychology, 27, 152-158.

Bagnara, S., Boles, D. B., Simion, F., \& Umiltà, C. (1982). Can an analytic/holistic dichotomy explain hemispheric asymmetries? Cortex, 18, 67-78.

Bagnara, S., Boles, D. B., Simion, F., \& Umiltà, C. (1983). Symmetry and similarity effects in the comparison of visual patterns. Perception \& Psychophysics, 34, 578-584.

BAMBer, D. (1969). Reaction times and error rates for samedifferent judgments of multidimensional stimuli. Perception \& Psychophysics, 6, 169-174.

Boles, D. B. (in press). Global versus local processing: Is there a hemispheric dichotomy? Neuropsychologia.

Conen, G. (1973). Hemispheric differences in serial versus parallel processing. Journal of Experimental Psychology, 97, 349-356.

Egeth, H. (1971). Laterality effects in perceptual matching. Perception \& Psychophysics, 9, 375-376.
Egeth, H. E., \& Epstein, J. (1972). Differential specialization of the cerebral hemispheres for the perception of sameness and difference. Perception \& Psychophysics, 12, 218-220.

Hellige, J. B., \& Webster, R. (1979). Right hemisphere superiority for initial stages of letter processing. Neuropsychologia, 17, 653-660.

Hock, H. S. (1973). The effects of stimulus structure and familiarity on same-different comparison. Perception \& Psychophysics, 14, 413-420.

Jonides, J. (1979). Left and right visual field superiority for letter classification. Quarterly Journal of Experimental Psychology, 31, 423-439.

KEPPEL, G. (1973). Design and analysis: $A$ researcher's handbook. Englewood Cliffs, N.J: Prentice-Hall.

Krueger, L. E. (1978). A theory of perceptual matching. Psychological Review, 85, 278-304.

Moscovitch, M., Scullion, D., \& Christie, D. (1976). Early vs. late stages of processing and their relation to functional hemispheric asymmetries in face recognition. Journal of Experimental Psychology: Human Perception \& Performance, 2, 401-416.

Nickenson, R. S. (1981). Context is important but it does not explain everything: A comment on "Matching performance and the similarity structure of the stimulus set" by Crist. Journal of Experimental Psychology: General, 110, 297-302.

Pattenson, K., \& Bradshaw, J. L. (1975). Differential hemispheric mediation of nonverbal visual stimuli. Journal of Experimental Psychology: Human Perception \& Performance, 1, 246-252.

Proctor, R. W. (1981). A unified theory for matching-task phenomena. Psychological Review, 88, 291-326.

Sergent, J. (1984). Hemispheric mediation of same-different judgments. Perception \& Psychophysics, 35, 592-595.

Shapiro, R. G., \& Krueger, L. E. (1983). Effect of similarity of surround on target-letter processing. Journal of Experimental Psychology: Human Perception and Performance, 9, 547-559.

TAYLOR, D. A. (1976). Holistic and analytic processes in the comparison of letters. Perception \& Psychophysics, 20, 187-190.

(Manuscript received May 11, 1984; accepted for publication May 23, 1984.) 